\title{
Turing stability of Ivlev-type predator model with a protection zone
}

\author{
Shuping Cao, Lina Zhang, Min Tian
}

\begin{abstract}
The Turing instability of Ivlev-type predator model of a protection zone is considered. Firstly, the stability of a positive equilibrium point in the corresponding ordinary different equation model, the existence of Hopf bifurcation are analyzed. Then the conditions of Turing instability are given by linearization analysis in the corresponding reaction diffusion model.
\end{abstract}

Index- Hopf bifurcation; Ivlev-type; protection zone; Turing instability.

\section{INTRODUCTION}

As we all know, the predator-prey dynamic system is one of the most important research topics in the mathematical and theoretical biology. In 1961, Ivlev[1] proposed the Ivlev type functional response term $1-\mathrm{e}^{-n x}$, which is widely used in invertebrates. Garvie[2] classified it as the Holling-II type functional response function. Turing instability is an important way to study spatial inhomogeneous pattern.

As early as 1905, Alan M Turing[3], a British mathematician, has explained the pattern of patterns in embryos, showing that in a diffusion system, diffusion destabilizes a steady state of uniformity under certain conditions and forms regular patterns in space, this is called turing instability or instability caused by diffusion. In recent years, many mathematicians and biologists have been interested in the predator-prey system of Ivlev, and great progress has been made in its research. The influence of diffusion on the stability, Hopf bifurcation and direction of the Ivlev-type predator-prey system is studied in paper [4].

In literature [5]-[7], Du et al. studied the effects of protected areas on L-V predator-prey model, Leslie predator-prey model and Holling-II predator-prey model. The turing instability of the Leslie-type and Ivlev-type self-diffusion models with protected areas is also studied in [8]-[11] .

In the paper, we discuss the self -diffusion model of predator-prey with protected area where $\Omega$ is a bounded domain with smooth boundary $\partial \Omega, v$ is the outward unit normal vector on the boundary; $r, a, m, n, \mu, b, c$ are normal numbers, $x, y$ are the degree functions of prey and predator population, respectively; $r, \mu$ are the intrinsic growth rates of $x$ and $y$, respectively; $n$ is the predator's rate of prey; $m$ is the number of protected areas; $a$ is the competition intensity

Shuping Cao, College of Mathematics and Statistics, Northwest Normal
University, Lanzhou Gansu 730070, China, phone number: 17326425052

Lina Zhang, College of Mathematics and Statistics, Northwest Normal University, Lanzhou Gansu 730070, China

Min Tian, College of Mathematics and Statistics, Northwest Normal University, Lanzhou Gansu 730070,China

$$
\left\{\begin{array}{l}
\mathrm{x}_{\mathrm{t}}-d_{1} \Delta x=x(r-a x)-\frac{(1-m)\left(1-e^{-n x}\right) y}{b+(1-m) x}, u \in \Omega, t>0 \\
y_{t}-d_{2} \Delta y=y\left(\mu-\frac{c y}{b+(1-m) x}\right), u \in \Omega, t>0 ; \\
\frac{\partial x}{\partial v}=\frac{\partial y}{\partial v}=0, u \in \partial \Omega, t>0 ; \\
x(u, 0)=x_{0}(u) \geq(\neq 0), y(u, 0)=y_{0}(u) \geq 0(\neq 0) .
\end{array}\right.
$$

between prey individual; $d$ is the semi saturation constant; normal number $d_{i}(i=1,2)$ is the diffusion coefficient corresponding to $x, y$. Homogeneous Neumann boundary indicates that the system is self- closing. That is to say, there is no population passing through the boundary; the initial value $x_{0}(u), y_{0}(u)$ is a non- negative smooth and non-constant zero function, where $1-e^{-n x}$ is Ivlev functional response term.

The turing instability of a predator-prey model with Ivlev-type functional response function and protected region term is studied by using qualitative analysis and bifurcation theory. The main contents are as follows: firstly, we discuss homogeneous systems in space without diffusion, analyze the local stability of positive equilibrium state and the existence of Hopf bifurcation; secondly, we study the conditions under which turing instability occurs for spatially inhomogeneous systems.

\section{STABILITY AND HOPF BIFURCATION OF SPATIALLY HOMOGENEOUS SYSTEMS}

The stability and Hopf bifurcation of homogeneous system study the following ODE System

$$
\left\{\begin{array}{l}
\frac{d x}{d t}=x(r-a x)-\frac{(1-m)\left(1-e^{-n x}\right) y}{b+(1-m) x}, t>0, \\
\frac{d y}{d t}=y\left(\mu-\frac{c y}{b+(1-m) x}\right), t>0, \\
x(t)=x_{0}>0, y(t)=y_{0}>0 .
\end{array}\right.
$$

it is easy to see that the system (2) has the following boundary equilibrium points:

(i)The trivial equilibrium $E_{0}(1,0)$, if the eigenvalue of Jacobian matrix at $E_{0}$ is $r, \mu$, then $E_{0}$ is saddle point unstable.

(ii)The semi-trivial equilibrium $E_{1}\left(\frac{r}{a}, 0\right)$, if the eigenvalue of jacobian matrix of model (2) at $E_{1}$ is $-r, \mu$, then $E_{1}$ is saddle point unstable.

(iii) $E^{*}=\left(x^{*}, y^{*}\right)$, where $x^{*}$ and $y^{*}$ satisfy the following algebraic equations 


$$
\begin{aligned}
& x(r-a x)-\frac{(1-m)\left(1-e^{-n x}\right) y}{b+(1-m) x}=0, \\
& \mu-\frac{c y}{b+(1-m) x}=0 .
\end{aligned}
$$

Then

$$
\frac{\mu x(r-a x)}{c(1-m)}=1-e^{-n x}
$$

then system (2) has a positive equilibrium if and only if the above equations have positive roots,

here $f(x)=\mu x(r-a x) \div c(1-m), g(x)=1-e^{-n x}$. It is easy to know that the intersection point of two curves in the first quadrant is the positive equilibrium point of the system. Let's discuss the monotonicity of $f(x)$ and $g(x), g^{\prime}(x)>0$, so $g(x)$ is monotonically increasing with respect to $x$. By a simple calculation, when $x \in\left(0, x_{1}\right), f^{\prime}(x)>0$, then $f(x)$ is monotonically increasing, when $x \in\left(x_{1}, \infty\right)$, $f^{\prime}(x)<0$, then $f(x)$ is monotonically decreasing and $f^{\prime}(x)=0$, here $x_{1}=\frac{r}{2 a}, f\left(\frac{r}{a}\right)=0$, obviously, $x_{1}<\frac{r}{a}$.

To sum up, the two curves must intersect in the first quadrant, indicating the existence of positive equilibrium. The following conclusions are easily obtained by using the linearization method.

\section{Theorem.1}

(i) when $\mu_{0}<0$, the positive equilibrium $E^{*}$ of system (2) is locally asymptotically stable.

(ii) when $0<\mu_{0}<\mu$, the positive equilibrium $E^{*}$ of system (2) is locally asymptotically stable.

(iii) when $\mu_{0}<0$, the positive equilibrium point $E^{*}$ of system (2) is unstable.

The jacobian matrix of system (2) at equilibrium point $E^{*}$ is

$$
J=\left(\begin{array}{cc}
\mu_{0} & -\frac{(1-m)\left(1-e^{-n x^{*}}\right)}{b+(1-m) x^{*}} \\
\frac{\mu^{2}(1-m)}{c} & -\mu
\end{array}\right)
$$

Here,

$$
\mu_{0}=r-2 a x^{*}+\frac{\mu(1-m)^{2}\left(1-e^{-n x^{*}}\right)}{c\left[b+(1-m) x^{*}\right]} .
$$

The characteristic equation of system (2) at $E^{*}$

$$
\lambda^{2}-T \lambda+D=0
$$

where

$$
\begin{aligned}
& T=\operatorname{tr} J=\mu_{0}-\mu, \\
& D=\operatorname{det} J=-\mu\left(r-2 a x^{*}\right) .
\end{aligned}
$$

(i)If $\mu_{0}<0$, so $T<0, D>0$, then the root real parts of the characteristic equation are all negative, so the positive equilibrium point $E^{*}$ is locally asymptotically stable.

(ii) If $0<\mu_{0}<\mu$, so $T<0, D>0$, then the root real parts of the characteristic equations are all less than zero. Therefore, the positive equilibrium point $E^{*}$ is locally asymptotically stable.

(iii) If $0<\mu_{0}$ and $\mu<\mu_{0}$, so $T>0, D>0$. So if the Jacobian Matrix and determinant has a pair of conjugate eigenvalues whose real parts are positive, then the equilibrium point $E^{*}$ is unstable.

The Hopf bifurcation of system (2) at $E^{*}$ is analyzed with $\mu$ as the bifurcation parameter. Suppose $0<\mu_{0}$ and $\mu<\mu_{0}$, let $\lambda=\alpha(\eta) \pm i \beta(\mu)$ be two roots of the characteristic equation and substitute it into the characteristic equation

$$
\left\{\begin{array}{l}
\alpha^{2}(\mu)-\beta^{2}(\mu)-\alpha(\mu)\left(\mu-\mu_{0}\right)-\mu\left(r-2 a x^{*}\right)=0 \\
2 \alpha(\mu) \beta(\mu)-\left(\mu_{0}-\mu\right) \beta(\mu)=0
\end{array}\right.
$$

when $\quad \mu=\mu_{0} \quad, \quad T=0 \quad$,then $2 \alpha(\mu) \beta(\mu)-\left(\mu_{0}-\mu\right) \beta(\mu)=0$. Derivative the left and right sides of the equation $2 \alpha(\mu) \beta(\mu)-\left(\mu_{0}-\mu\right) \beta(\mu)=0$, so $\alpha^{\prime}(\mu)=-\frac{1}{2}<0$, then the condition of transversality holds. According to the Poincare-Andronov-Hopf bifurcation theorem, when the parameter $\mu$ changes and passes through the critical value $\mu_{0}$, system (2) produces Hopf bifurcation at $E^{*}$.

\section{DYNAMICS OF THE ODE MODEL}

Based on the discussion of ODE system, the stability of the space homogeneous positive equilibrium solution $\left(x^{*}, y^{*}\right)$ of one-dimensional $\Omega=(0, l)$ system with diffusion is further discussed

$$
\left\{\begin{array}{l}
x_{t}-d_{1} x^{\prime \prime}=x(r-a x)-\frac{(1-m)\left(1-e^{-n x}\right) y}{b+(1-m) x}, x \in(0, l), t>0 \\
y_{t}-d_{2} y^{\prime \prime}=y\left(\mu-\frac{c y}{b+(1-m) x}\right), y \in(0, l), t>0 \\
\frac{\partial x}{\partial v}=\frac{\partial y}{\partial v}=0, u=0, l \\
x(u, 0)=x_{0}(u), y(u, 0)=y_{0}(x), x \in(0, l)
\end{array}\right.
$$

Define the real value Soblev space $X=\left\{(x, y) \in H^{2}(0, l) \times H^{2}(0, l):\left.\left(x_{u}, y_{u}\right)\right|_{u=0, l}=0\right\}$ and complex space $X(c)=X+X i=\{x+i y: x, y \in X\}$. Easy to know, the linear eigenvalue problem

$$
\left\{\begin{array}{l}
-\phi_{u u}=\lambda \phi, u \in(0, l), \\
\phi_{u}=0, u=0, l
\end{array}\right.
$$

Has simple eigenvalu $\mu_{k}=k^{2}, k \in N$, and $\varphi_{k}=\cos k x, k \in N$ is the principal eigenfunction corresponding to $\mu_{k}$. Simple analysis shows that the trivial and semi-trivial equilibrium points of system (3) always exist and have the same stability as system (2).

The linearized equation of system (3) at $E^{*}$ has the following form

$$
\left(\begin{array}{l}
x_{t} \\
y_{t}
\end{array}\right)=L\left(\begin{array}{l}
x \\
y
\end{array}\right)=D \Delta\left(\begin{array}{l}
x \\
y
\end{array}\right)+J\left(\begin{array}{l}
x \\
y
\end{array}\right)
$$


Here, $D=\operatorname{diag}\left(d_{1}, d_{2}\right)$, Here $J$ is given in the proof of Theorem.1.

For $k \in N$, let

$$
L_{k}=\left(\begin{array}{cc}
-d_{1} k^{2}+\mu_{0} & -\frac{(1-m)\left(1-e^{-n x}\right)}{b+(1-m) x} \\
\frac{\mu^{2}(1-m)}{c} & -d_{2} k^{2}-\mu
\end{array}\right)
$$

the characteristic equation corresponding to $L_{k}$ is

$$
B_{k}(\lambda):=\lambda^{2}-T_{k} \lambda+D_{k}=0
$$

Where

$$
\begin{aligned}
& T_{k}=-\left(d_{1}+d_{2}\right) k^{2}+\mu_{0}-\mu, \\
& D=d_{1} d_{2} k^{4}+\left(d_{1} \mu-d_{2} \mu_{0}\right) k^{2}-\mu\left(r-2 a x^{*}\right) .
\end{aligned}
$$

we assume $\mu_{0} \leq 0$, it is easy to see $T_{k}<0, D_{k}>0$. Then both roots of $B_{k}(\lambda)$ have negative real parts, and the following theorem is easily obtained.

\section{Theorem.2}

If $\mu_{0} \leq 0$, then the positive equilibrium $E^{*}\left(x^{*}, y^{*}\right)$ of the system (3) is locally asymptotically stable.

If $\mu_{0}>0$ and $\mu_{0} \leq \mu$, the internal equilibrium solution of ODE system is locally asymptotically stable.

The turing stability of the spatial homogeneous solution of the diffusion system (3) is studied under the above conditions.

It is well known that the coexisting equilibrium solution of system (3) is unstable if at least one root has a positive real part. Note that when $\mu>\mu_{0}>0, T_{k}<0$, then system (3) has no imaginary root whose real part is positive. For simplicity, define quadratic polynomials with respect to $k^{2}$ and determine the sign

$$
\varphi\left(k^{2}\right)=d_{1} d_{2} k^{4}+\left(d_{1} \mu-d_{2} \mu_{0}\right) k^{2}-\mu\left(r-2 a x^{*}\right) .
$$

If $\varphi\left(k^{2}\right)<0$, then the system (3) has one positive and one negative root. $\Lambda\left(d_{1}, d_{2}\right)=d_{1} \mu-d_{2} \mu_{0}<0$, for Some $k^{2}$ to be $\varphi\left(k^{2}\right)<0$, there must be a minimum value of $\varphi\left(k^{2}\right)$ in $k^{2}=k_{\min }^{2}$

$$
\min _{k} \varphi\left(k^{2}\right)=-\mu\left(r-2 a x^{*}\right)-\frac{\left(d_{1} \mu-d_{2} \mu_{0}\right)^{2}}{4 d_{1} d_{2}}<0
$$

where

$$
k_{\min }^{2}=-\frac{d_{1} \mu-d_{2} \mu_{0}}{2 d_{1} d_{2}}
$$

let $\theta=\frac{d_{2}}{d_{1}}$.

$$
\begin{array}{r}
\Lambda^{*}\left(d_{1}, d_{2}\right):=\left(d_{1} \mu-d_{2} \mu_{0}\right)^{2}-4 d_{1} d_{2}\left[-\mu\left(r-2 a x^{*}\right)\right] \\
=\mu_{0}{ }^{2} d_{2}{ }^{2}+2 d_{1} d_{2} \mu\left[-\mu_{0}+2\left(r-2 a x^{*}\right)\right]+\mu^{2} d_{1}{ }^{2} .
\end{array}
$$

let $\Lambda^{*}\left(d_{1}, d_{2}\right)=0, \Lambda\left(d_{1}, d_{2}\right)=0$.

Simplified

$$
\begin{gathered}
\mu^{2}+2 \theta \mu\left[-\mu_{0}+2\left(r-2 a x^{*}\right)\right]+\mu_{0}^{2} \theta^{2}=0, \\
\theta=\frac{\mu}{\mu_{0}}=\theta^{*}
\end{gathered}
$$

We note that

$$
D=-\mu\left(r-2 a x^{*}\right)>0, r-2 a x^{*}<0,
$$

then

$4 \mu^{2}\left[-\mu_{0}+2\left(r-a x^{*}\right)\right]^{2}-4 \mu^{2} \mu_{0}^{2}=16 \mu^{2}\left(r-2 a x^{*}\right)\left[-\mu_{0}+\left(r-2 a x^{*}\right)\right]>0$.

so $\Lambda^{*}\left(d_{1}, d_{2}\right)=0$ has the following two position roots

$$
\begin{aligned}
& \theta_{1}=\frac{\left.\left\{\mu\left[-\mu_{0}+2\left(r-2 a x^{*}\right)\right]+2 \mu \sqrt{\left(r-2 a x^{*}\right)\left[-\mu_{0}+\left(r-2 a x^{*}\right)\right.}\right]\right\}}{\mu_{0}^{2}}, \\
& \theta_{2}=\frac{\left\{\mu\left[-\mu_{0}+2\left(r-2 a x^{*}\right)\right]-2 \mu \sqrt{\left(r-2 a x^{*}\right)\left[-\mu_{0}+\left(r-2 a x^{*}\right)\right.}\right]}{\mu_{0}^{2}} .
\end{aligned}
$$

We obtain $0<\theta_{2}<\theta^{*}<\theta_{1}$, when $\frac{d_{2}}{d_{1}}>\theta$, $\min _{k} \varphi\left(k^{2}\right)<0, \Lambda\left(d_{1}, d_{2}\right)<0$.So $\left(x^{*}, y^{*}\right)$ is unstable, which means turing instability occurs.

Based on the above discussion, the following conclusions about diffusion-induced instability are drawn.

\section{Theorem.3}

If $\mu_{0}>0$ and $\mu_{0}<\mu$ hold, the coexisting steady-state solutions is also stable for local system (2), so there's an unbounded region $\Gamma=\left\{\left(d_{1}, d_{2}\right): d_{1}>0, d_{2}>0, d_{2}>d_{1} \theta_{1}\right\}$, for $\theta_{1}>0$. when $\left(d_{1}, d_{2}\right) \in \Gamma$, the equilibrium solution of system (3) is turing instability.

\section{CONCLUSION}

The stability of the positive solution of the reaction-diffusion system is analyzed by means of linearization method. It is shown that under certain conditions, linear self-diffusion can cause a change in the stability of the model, that is, to produce Turing instability in classical sense.

\section{REFERENCES}

[1] V. S. Ivlev, Douglas Scott, Saul B. Sailu. Experimental Ecology of the Feeding of Fishes.[J]. Quarterly Review of Biology, 1964.

[2] M .R. Garvie. Finite-difference schemes for reaction diffusion equations modeling predator-prey interaction in MATLAB $[\mathrm{J}]$. Bulletin of Mathematical Biology, 2007, 69(3): 931-956.

[3] A. M. Turing. The Chemical Basis of Morphogenesis[J]. Bulletin of Mathematical Biology, 1952, 237(641):37-72.

[4] X .Wang, J. Wei, J. Jun, Diffusion-driven stability and bifurcation in a predator-prey system with Ivlev-type functional response [J]. Applicable Analysis, 2013,92(4): 752-775.

[5] Y. Du, X. Liang, A diffusive competition model with a protection zone[J]. Journal of Differential Equations, 2008, 244(1):61-86.

[6] Y. Du, R .Peng, M .Wang, Effect of a protection zone in the diffusive Leslie predator-prey model [J]. Journal of Differential Equations, 2009, 246(10):3932-3956.

[7] Y .Du, J. Shi, A diffusive predator-prey model with a protection zone[J] Journal of Differential Equations, 2006, 229(1):63-91.

[8] X . Chang, J. Wei, Stability and Hopf bifurcation in a diffusive predator-prey system incorporating a prey refuge [J]. Mathematical Biosciences and Engineering, 2013, 10(4):979-996.

[9] R. Yang, J .Wei. Stability and bifurcation analysis of a diffusive prey-predator system in Holling type III with a prey refuge $[\mathrm{J}]$. Nonlinear Dynamics, 2015, 79(1):631-646.

[10] H. B. Shi, S. Ruan, Y. Su, et al. Spatiotemporal Dynamics of a Diffusive Leslie-Gower Predator-Prey Model with Ratio-Dependent Functional Response[J]. International Journal of Bifurcation and Chaos, 2015, 25(05).

[11] X .Guan, W .Wang, Y. Cai, Spatiotemporal dynamics of a Leslie-Gower predator-prey model incorporating a refuge [J]. Nonliner Anal. Real, 2011, 12(4): 2385-2395 